\title{
A finger on the pulse of Wnt receptor signaling
}

\author{
Bryan T MacDonald ${ }^{1}, \mathrm{Xi} \mathrm{He}^{1}$ \\ ${ }^{I}$ The F. M. Kirby Neurobiology Center, Boston Children's Hospital, Department of Neurology, Harvard Medical School, Boston, \\ MA 02115, USA \\ Cell Research (2012) 22:1410-1412. doi:10.1038/cr.2012.91; published online 12 June 2012
}

In a recent issue of Nature, Hao et al. report an unexpected link between the secreted stem cell factor/ Wnt agonist $R$-spondin and Wnt receptors through the transmembrane ZNRF3 protein, a RING finger ubiquitin ligase. ZNRF3 acts to turn over Frizzled and LRP6 receptors. Rspondin binds to ZNRF3, in addition to transmembrane LGR $4 / 5$ receptors, to antagonize degradation of the Wnt receptors by ZNRF3, thereby resulting in increased Frizzled and LRP6 levels and a greater Wnt response.

Signaling by the Wnt family of secreted morphogens has central roles in embryogenesis, homeostasis, pathogenesis, and regeneration [1]. Wnt proteins engage the Frizzled (FZ) family of serpentine receptors and various co-receptors including the LDL receptor-related protein 6 (LRP6) [1]. In the canonical Wnt pathway, Wntinduced FZ-LRP6 complex formation activates $\beta$-catenin (a transcriptional coactivator)-dependent gene expression [1]. R-spodin (Rspo) proteins are four related and secreted Wnt agonists that facilitate $\mathrm{Wnt} / \beta$-catenin activation in stem cells and many developmental events in vertebrates $[2,3]$. But how Rspo proteins act mechanistically has been enigmatic.

Rspo proteins have gained promi-

Correspondence: Xi He

E-mail: xi.he@childrens.harvard.edu nence as potent mitogens for intestinal stem cells [3], whose self-renewing proliferation is powered by $\mathrm{Wnt} / \beta$-catenin signaling [4]. Rspo synergizes with low levels of Wnt to enhance phosphorylation and activation of LRP6, and phosphorylation of Dishevelled [5], a FZ downstream partner and indicator of FZ activation, suggesting that Rspo acts through the Wnt receptors. An important feature of Rspo action is its dependence on Wnt, because Rspo effect is blocked by secreted Wnt antagonists (such as DKK1 and Sfrps) $[2,5,6]$ and by chemical inhibition of Wnt biogenesis [4]. How Rspo proteins serve as Wnt agonists had been debated, as conflicting evidence existed for or against Rspo binding to LRP6, FZ or another protein (Kremen) [2, 5-7]. This debate was quieted to a large degree by publications that identified leucine-rich $G$ protein coupled receptors 4 and 5 (LGR4/5) as Rspo receptors $[8,9]$.

LGR4, LGR5 and the related LGR6 form a unique group of serpentine receptors with a large extracellular domain containing leucine-rich repeats [10]. LGR5 had already gained fame as a marker for proliferating intestinal stem cells and is a Wnt target gene [10]. LGR4/5 through their amino terminal region bind to Rspo with high affinity, and their knockdown via siRNAs/shRNAs diminished activation of $\beta$-catenin signaling by Rspo, but not Wnt, treatment $[8,9,11]$. Importantly, genetic deletion of Lgr $4 / 5$ in mice abolishes self-renewal and Wnt-responsive gene expression in intestinal stem cells [9]. A FZ-LRP6-LGR4/5 complex has been observed in the presence of Rspo plus Wnt $[9,12]$ (Figure 1), leading to a view that while the FZ-LRP6 complex mediates $\mathrm{Wnt} / \beta$-catenin signaling, a supercomplex of FZ-LRP6-LGR4/5 mediates Rspo enhancement of the Wnt pathway (Figure 1). But how LGR4/5 (and likely LGR6), which may not act like typical G-protein coupled receptors (GPCRs) [8, 9], enhance FZ-LRP6 signaling remains unknown. Hao et al. [13] shed light on this important question and beyond by demonstrating a role of ZNRF3 as an additional Rspo receptor that regulates FZ and LRP6 levels on the plasma membrane.

Hao et al. [13] designed a clever computation screen to identify new Wnt target genes that correlate with expression of Axin2, an established Wnt target gene. This rationale is logical as many Wnt regulators, such as Rspo and LGR5 (pathway enhancers), and DKK1 and Axin2 (pathway antagonists), are directly or indirectly activated by Wnt signaling [1]. From the screen, the single-span transmemebrane RING finger ubiquitin ligases ZNRF3 and related RNF43 emerged as top candidates. Hao et al. demonstrated that both ZNRF3 and RNF43 were indeed induced by Wnt stimulation and upregulated in colon cancers that are known for high $\beta$-catenin signaling. Hao et al. defined ZNRF3 and RNF43 as inhibitors of the Wnt pathway using knockdown and 
A Without Rspo

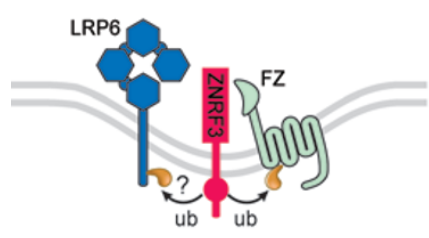

LRP6 and FZ Turnover
B With Rspo plus Wnt

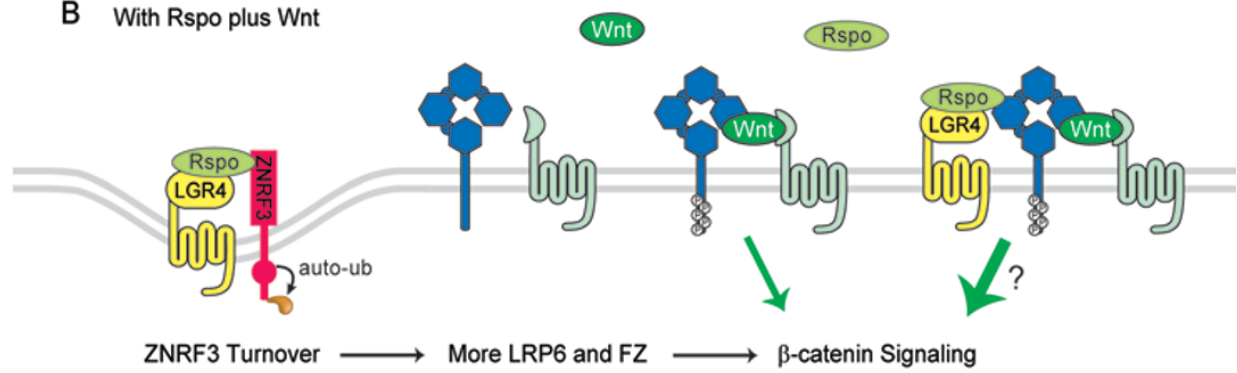

Figure 1 (A) ZNRF3 promotes turnover of FZ and LRP6 via acting as a ubiquitin ligase. (B) Rspo binds to both ZNRF3 and LGR4, which promotes ZNRF3 turnover likely through its autoubiquitination, thereby stabilizing FZ and LRP6 for a greater Wnt response. Whether LGR4 has additional signaling function in the FZ-LRP6 complex is unknown. Rspo stabilization of FZ also enhances Wnt/PCP signaling (not depicted).

overexpression methods. Knockdown of ZNRF3 increased Wnt-induced reporter and cytosolic $\beta$-catenin levels, and this increase was abrogated when Wnt biogenesis was inhibited, indicating that signaling by endogenous Wnt ligands was elevated when ZNRF3 was depleted. A ZNRF3 mutant lacking the intracellular RING finger ubiqutin ligase domain behaved in a dominant negative manner, elevating Wnt signaling. ZNRF3 knockdown or inhibition by the dominant negative ZNRF3 mutant increased phosphorylation of LRP6 and Dishevelled, suggesting an involvement of both LRP6 and FZ [13]. Importantly depletion or blocking ZNRF3 function resulted in increased FZ and LRP6 levels on the plasma membrane due to extended receptor half-life. A previous study showed that FZ ubiquitination and degradation through the lysosomal pathway negatively regulates Wnt responsiveness [14]. Demonstrating that ZNRF3 likely acts as a FZ ubiquitin ligase, Hao et al. showed that FZ was ubiquitinated and degraded in a ZNRF3dependent manner that depended on the RING finger domain, and a FZ mutant engineered to lack lysines for ubiquitin attachment was resistant to ubiquitination and degradation by ZNRF3. Further, ZNRF3 was found to coimmunoprecipitate with FZ and LRP6. Therefore ZNRF3, and likely RNF43, negatively regulate Wnt receptor stability through ubiquitination-mediated degradation.

Hao et al. [13] prudently noticed that Rspo increased FZ and LRP6 levels on the plasma membrane while decreased FZ ubiquitination, and therefore phenocopied the effects of knockdown/ blocking of ZNFR3. This prompted the authors to explore whether Rspo acts to inhibit ZNRF3. Strikingly, they found that Rspo bound to ZNRF3 extracellular domain (ECD), and this interaction was prevented by antibodies against ZNRF3-ECD or by a mutation of a conserved proline in ZNRF-ECD. Further supporting Rspo-ZNRF3 binding, ZNRF3-ECD (anchored on the membrane) could act as a decoy that inhibited signaling by Rspo but not Wnt, and prevented Rspo-induced increase of FZ on the plasma membrane. LGR4 as a Rspo receptor was required for Rspo-induced inhibition of FZ ubiquitination and elevation of $\mathrm{FZ}$ and LRP6 levels, as these Rspo effects were diminished via LGR4 knockdown. Hao et al. further observed a Rspo-induced ZNRF3-LGR4 complex formation, which accompanied Rspo-triggered removal of ZNRF3 from the plasma membrane (Figure 1). Indeed LGR4 and Rspo-ZNRF3 binding were both required, while forced LGR4-ZNRF3 dimerization was sufficient, for ZNRF3 clearance, which additionally required the RING finger domain of ZNRF3 (i.e., autoubiquitination via its own ubiquitin ligase activity) [13] (Figure 1). There- fore Hao et al. proposed a new model in which Rspo binds to LGR4 and ZNRF3, resulting in the removal of ZNRF3 from, and the consequent stabilization of, the Wnt receptors (Figure 1).

Like Rspo genes, ZNRF3 and RNF43 are conserved in vertebrates. Hao et al. [13] confirmed that Znrf3 has a negative role in $\mathrm{Wnt} / \beta$-catenin signaling in dorsal axis determination and anteriorposterior patterning in Xenopus and/or zebrafish embryos. The observation that Rspo stabilizes FZ proteins offers a satisfying explanation for Rspo regulation of non-canonical Wnt planar cell polarity (PCP) signaling $[5,15]$, in which FZ has a central role. Indeed the authors demonstrated an involvement of Znrf3 in $\mathrm{Wnt} / \mathrm{PCP}$ signaling in gastrulation in zebrafish embryos. The authors went further and generated $\mathrm{Znrf}^{-/-}$mice, which are perinatal lethal. Preliminary characterization suggested multiple developmental defects, including elevated Axin2 expression and abnormal lens development (which are consistent with excessive $\beta$-catenin signaling) and neural tube closure anomaly (which is associated with defective PCP signaling). It is conceivable that in mouse embryogenesis Znrf3 and Rnf43 may have partially redundant functions, which may be observable only in double knockout mutants.

Hao et al.'s comprehensive study has several broad implications: (i) it identifies ZNRF3 and RNF43 as novel 
transmembrane ubiquitin ligases for degradation of FZ and possibly LRP6 receptors; (ii) it reveals a new negative feedback loop as ZNRF3 and RNF43 are induced by Wnt signaling; (iii) most interestingly it demonstrates that Rspo is an antagonistic ligand for ZNRF3 by inducing Rspo-ZNRF3-LGR4 complex formation, which causes clearance of ZNRF3 from the plasma membrane thereby stabilizing FZ and LRP6 receptors. This study provides significant insights into the mechanism by which Rspo proteins act as Wnt agonists in $\beta$-catenin and PCP pathways during vertebrate embryogenesis, and has ramifications for understanding stem cell biology, cancer and other diseases. Loss-of-function mutations in RNF43 have been reported in pancreatic cancer patients, indicating that RNF43 may act as a tumor suppressor [16], and RSPO3 and ZNRF3 are among loci associated with risk of metabolic disorders [17].

Many questions remain to be addressed. Several transmembrane complexes have been proposed, including FZ-ZNRF3 (in the absence of Rspo), LGR4/5-ZNRF3 (in the presence of Rspo) [13], and a FZ-LRP6-LGR4/5 supercomplex (in the presence of Wnt plus Rspo) [9, 12] (Figure 1), but the relationships among these complexes are obscure. Do LGR $4 / 5$ act solely to antagonize ZNRF3? Or do LGR4/5, which resemble GPCRs, have additional signaling function in receptor complexes for $\beta$-catenin and PCP pathways? How does ZNRF3 act as a FZ ubiquitin ligase that is inhibited by Rspo? The fact that two ZNRF3 mutants that lack the RING finger domain only and the entire intracellular domain, respectively, behave in opposite manners [13] implies the need to further characterize ZNRF3 molecularly and to identify its additional binding partners. Related to these issues are the apparently contradictory observations of Rspo stabilizing FZ on the plasma membrane [13] versus Rspo promoting FZ endocytosis via the clathrin pathway, and the importance (or not) of FZ endocytosis in Rspo responses [8, $11,12]$. Investigation into these questions will further our understanding of Rspo and Wnt signaling in development, cancer and drug discoveries.

\section{Acknowledgments}

X H acknowledges support by NIH (grants RO1 GM057603, GM057603S1, GM074241, and AR060359) and by the Boston Children's Hospital Intellectual and Developmental Disabilities Research Center (P30 HD-18655).

\section{References}

1 MacDonald BT, Tamai K, He X. Wnt/ beta-catenin signaling: components, mechanisms, and diseases. Dev Cell 2009; 17:9-26.

2 Kazanskaya O, Glinka A, del Barco Barrantes I, et al. R-Spondin2 is a secreted activator of Wnt/beta-catenin signaling and is required for Xenopus myogenesis. Dev Cell 2004; 7:525534.

3 Kim KA, Kakitani M, Zhao J, et al. Mitogenic influence of human R-spondin 1 on the intestinal epithelium. Science 2005; 309:1256-1259.

4 Sato T, van Es JH, Snippert HJ, et al. Paneth cells constitute the niche for Lgr5 stem cells in intestinal crypts. $\mathrm{Na}$ ture 2011; 469:415-418.

5 Wei Q, Yokota C, Semenov MV, et al. $\mathrm{R}$-spondin1 is a high affinity ligand for LRP6 and induces LRP6 phosphorylation and beta-catenin signaling. $J$ Biol Chem 2007; 282:15903-15911.

6 Nam JS, Turcotte TJ, Smith PF, Choi $\mathrm{S}$, Yoon JK. Mouse cristin/R-spondin family proteins are novel ligands for the Frizzled 8 and LRP6 receptors and activate beta-catenin-dependent gene expression. J Biol Chem 2006; 281:13247-13257.

7 Binnerts ME, Kim KA, Bright JM, et al. R-Spondin1 regulates Wnt signal- ing by inhibiting internalization of LRP6. Proc Natl Acad Sci USA 2007; 104:14700-14705.

8 Carmon KS, Gong X, Lin Q, Thomas A, Liu Q. R-spondins function as ligands of the orphan receptors LGR4 and LGR5 to regulate Wnt/beta-catenin signaling. Proc Natl Acad Sci USA 2011; 108:11452-11457.

9 de Lau W, Barker N, Low TY, et al. Lgr5 homologues associate with Wnt receptors and mediate $\mathrm{R}$-spondin signalling. Nature 2011; 476:293-297.

10 Barker N, Clevers H. Leucine-rich repeat-containing G-protein-coupled receptors as markers of adult stem cells. Gastroenterology 2010; 138:16811696.

11 Glinka A, Dolde C, Kirsch N, et al. LGR4 and LGR5 are R-spondin receptors mediating Wnt/beta-catenin and Wnt/PCP signalling. EMBO Rep 2011; 12:1055-1061.

12 Carmon KS, Lin Q, Gong X, Thomas A, Liu Q. LGR5 interacts and cointernalizes with Wnt receptors to modulate Wnt/beta-catenin signaling. Mol Cell Biol 2012; 32:2054-2064.

13 Hao HX, Xie Y, Zhang Y, et al. ZNRF3 promotes Wnt receptor turnover in an R-spondin-sensitive manner. Nature 2012; 485:195-200.

14 Mukai A, Yamamoto-Hino M, Awano $\mathrm{W}$, et al. Balanced ubiquitylation and deubiquitylation of Frizzled regulate cellular responsiveness to $\mathrm{Wg} / \mathrm{Wnt}$. EMBO J 2010; 29:2114-2125.

15 Ohkawara B, Glinka A, Niehrs C. Rspo3 binds syndecan 4 and induces Wnt/PCP signaling via clathrin-mediated endocytosis to promote morphogenesis. Dev Cell 2011; 20:303-314.

$16 \mathrm{Wu}$ J, Jiao Y, Dal Molin M, et al. Whole-exome sequencing of neoplastic cysts of the pancreas reveals recurrent mutations in components of ubiquitindependent pathways. Proc Natl Acad Sci USA 2011; 108:21188-21193.

17 Heid IM, Jackson AU, Randall JC, et al. Meta-analysis identifies 13 new loci associated with waist-hip ratio and reveals sexual dimorphism in the genetic basis of fat distribution. Nat Genet 2010; 42:949-960. 